\title{
Reported incidence and treatment of dermatophytosis in children in general practice: a comparison between 1987 and 2001
}

\author{
R. S. A. Mohammedamin · J. C. van der Wouden $\cdot$ S. Koning $\cdot$ \\ F. G. Schellevis · L. W. A. van Suijlekom-Smit · B. W. Koes
}

Received: 23 April 2007 / Accepted: 3 September 2007/Published online: 23 September 2007

(C) Springer Science+Business Media B.V. 2007

\begin{abstract}
Introduction Dermatophytosis is a common skin infection in children. Although the epidemiology is relatively unknown it is becoming a major health problem in some countries.We determine the incidence and management of dermatophytosis in Dutch general practice in 1987 and 2001.

Methods We used data of all children aged 0-17 years derived from two national surveys performed in Dutch general practice in 1987 and 2001 respectively. All diagnoses, prescriptions and referrals were registered over a 12 months period by the participating general practitioners (GPs), 161 and 195 respectively. Data were stratified for socio-demographic characteristics.
\end{abstract}

R. S. A. Mohammedamin · J. C. van der Wouden ( $\square)$. S. Koning · B. W. Koes

Department of General Practice, Room Ff304, Erasmus MC-University Medical Center, P. O. Box 2040, 3000 DR Rotterdam, The Netherlands

e-mail: j.vanderwouden@erasmusmc.nl

F. G. Schellevis

Department of General Practice, Netherlands Institute for Health Services Research (NIVEL), Free University, Utrecht, Amsterdam, The Netherlands

L. W. A. van Suijlekom-Smit

Department of Paediatrics, Sophia Children's Hospital, Erasmus MC-University Medical Center, Rotterdam, The Netherlands
Results Compared to 1987, in 2001 the total reported incidence rate of dermatophytosis in children in general practice increased from 20.8 [95\% CI 18.9-22.8] to 24.6 [95\%CI 23.5-25.7] per 1,000 person years. Infants ( $<1$ year), girls, children in rural areas and children of non-western immigrants more often consulted the GP for dermatophytosis in 2001. In both surveys GPs treated the majority of children with dermatophytosis with topical drugs, especially with azoles.

Conclusions The reported incidence rate of dermatophytosis in children in general practice increased; however it is unclear whether this is a consequence of an increasing prevalence in the population or a changing help seeking behaviour. GPs generally follow the national guideline for the treatment of dermatophytosis in children.

Keywords Dermatophytosis - Children · General practice $\cdot$ Incidence $\cdot$ Management

\section{Introduction}

Superficial fungal skin infections (dermatophytosis) caused by dermatophytes are known as tinea and will have a specific name depending on body location, as in tinea capitis, tinea corporis, or tinea pedis [1]. Dermatophytoses are common in children [2-4].

Little data is available about the epidemiology of dermatophytosis in children in the population. Most 
studies in this field dealt with adults or with a specific form of dermatophytosis. The few surveys performed in children reported a prevalence that varies from $2.5 \%$ to $15.2 \%$ [5-7] and differ from country to country. Tinea capitis is predominantly a disease of children, especially under 12 years of age, and rare in adults [2-4, 7-12]. The frequency of tinea capitis is declining in many developed nations; however in the United Kingdom and the United States it is becoming a major public health problem, and Afro-Caribbean children are particularly affected [5, 9-13]. Data about the other forms of dermatophytosis in children are relatively limited available.

Initial analysis by Otters et al showed that the incidence rate of dermatophytosis in children in Dutch general practice has increased between 1987 and 2001 [14]. It is unclear whether this increase reflected an increase of the incidence in the population and is related to socio-demographic characteristics. Knowledge about the factors contributing to the increasing incidence might improve the care for patients with dermatophytosis in general practice and its prevention.

Both topical and oral treatments have been proven to be effective, though not for all dermatophytoses [15-17]. However, in a British study only $7 \%$ of children had received appropriate treatment for tinea capitis before referral to dermatology practice [11]. The evaluation of management of dermatophytosis in children in general practice is therefore important. In 1997, the Dutch College of General Practitioners issued a clinical guideline for the diagnosis and treatment of dermatophytosis for all ages [18]. We do not have a clear insight in the degree of application of this guideline in children.

In the present study we compared the results of two consecutive surveys in general practice performed in 1987 and 2001 respectively and aimed to answer the following research questions:

- What was the reported incidence rate of dermatophytosis in children aged $0-17$ in general practice in 1987 and in 2001?

- Were these incidence rates related to sociodemographic characteristics?

- How did the GP manage dermatophytosis (prescription, referral) and did this change between 1987 and 2001 ?

\section{Methods}

We used data from the first and second Dutch national surveys of general practice, which were performed by the Netherlands Institute for Health Services Research (NIVEL) in 1987 and 2001. Each survey included a representative sample of the Dutch population. In the Netherlands, general practices have a fixed list size, all inhabitants are listed in a general practice, and GPs have a gate-keeping role, for adults as well as for children. Usually, the first contact with health care, in a broad sense, is the contact with the general practitioner. In this respect the Dutch health care structure did not change between 1987 and 2001.

In 1987 a sample, non-proportionally stratified by region, degree of urbanisation and distance to nearest hospital, of 161 GPs in 103 practices was selected randomly to participate in the first national survey [19]. With respect to age and gender the participating GPs and practices were representative of Dutch GPs and practices in 1987. The GPs were divided into four groups, and each group used registration forms to register data (e.g. diagnosis, prescription and referrals) on all contacts between patient and practice during one of four consecutive 3-month periods. Baseline characteristics such as age and gender were derived from patient records. Other socio-demographic characteristics such as socio-economic status (SES) and ethnic origin were obtained by a questionnaire and filled out by parents, or by the children themselves if they were older than 12 years (response rate 91.2\%). SES was based on the father's occupation, which was categorized into five classes "non-manual work high (class I)", "non-manual work middle (class II)", "non-manual low and farmers (class III)", "manual work high/middle (class IV)" and "manual work low (class V)". Ethnic origin was derived from the reported country of birth of either parent. If either parent was born in Turkey, Africa, Asia (except Japan and Indonesia) and Central or South America, their children were considered to be children of non-Western origin (in accordance with the classification of Statistics Netherlands). All other children were defined as Western. The degree of urbanization was derived from the general practice's postal code and categorized into four classes 'under 30,000 inhabitants', '30,00050,000 inhabitants', 'over 50,000 inhabitants' and 
'the three large Dutch cities Amsterdam, Rotterdam and The Hague'. The Netherlands were divided into a Northern, Central and Southern region. Season was divided into four categories: spring was defined as months April-June, summer as July-September, autumn as October-December and winter as January-March. The diagnoses made by the GPs were coded afterwards by clerks using the International Classification of Primary Care (ICPC), a classification commonly used in primary care [20].

In 2001, data about all physician-patient contacts over 12 months were derived from the electronic medical records of all listed patients in 104 practices (195 GPs) [21]. The GPs registered data on diagnosis, prescriptions and referrals, and coded the diagnosis themselves using the ICPC. Patient demographic characteristics such as age and gender were derived from the GPs' computerized patient files. As in 1987, SES and ethnicity were obtained by a questionnaire (response rate $76 \%$ ). Degree of urbanization, region and season were defined as in 1987.

In both surveys each contact with the GP was defined as one consultation. All health problems presented within one consultation were recorded separately. Both surveys were episode orientated, meaning that a consultation on a new health problem marked the beginning of a new episode. If there were multiple consultations in a single episode, the diagnosis made during the last consultation was regarded as the episode-diagnosis. In both surveys all prescriptions were coded according to the AnatomicalTherapeutical-Chemical (ATC) classification, as developed by WHO (www.whocc.no/atcddd). Concerning referrals, the GPs registered the indication and specialism of referral. In 2001 we excluded data from nine practices from the analyses, mainly because of technical problems with registration. The Dutch guideline allows the GP to diagnose dermatophytes without a $\mathrm{KOH}$ preparation or culture in most cases and we do not know how certain the GP was of this diagnosis [18].

\section{Ethical approval}

The study was carried out according to Dutch legislation on privacy. The privacy regulation of the study was approved by the Dutch Data Protection Authority. According to Dutch legislation, obtaining informed consent is not obligatory for observational studies.

\section{Data-analysis}

This study analyzed data from both surveys for children aged 0-17 years presenting with dermatophytosis which was coded as S74 (ICPC). Because of an underrepresentation of deprived areas, the 1987 survey population was weighted to the Dutch population of 1987. Incidence rates were calculated by dividing the weighted number of reported new episodes (numerator) by the study population at risk (denominator).

For 2001 we calculated the incidence rate of dermatophytosis by dividing the total number of reported new episodes (numerator) by the average study population at risk, the mid-time population (denominator). The mid-time population was calculated as the mean of all listed patients of all participating GPs, aged 0-17 years, at the beginning and at the end of the registration period. Data were stratified by age, gender, urbanization level, region, season, SES and ethnic origin. Incidence rates per 1,000 person-years and $95 \%$ confidence intervals were calculated assuming a Poisson distribution. In both surveys, we only included the first new episode for every child. Thus we excluded two recurrent episodes in 1987 and 77 in 2001.

Prescriptions and referrals were expressed as proportions of all new episodes.

\section{Results}

Study populations in 1987 and 2001

The study population in 1987 consisted of 86,577 children aged 0-17 years yielding 21,644 person years. These children had 559 contacts concerning dermatophytosis which contributed to 450 episodes; $85.3 \%$ of these episodes included a single contact with the GP. In 2001 there were 87,952 children yielding 81,716 person-years. These children had 2,318 contacts concerning dermatophytosis which contributed to 2,007 episodes; $88.2 \%$ of these episodes consisted of only one contact with the GP. 
Incidence rate

Table 1 shows the distribution of reported incidence rates of dermatophytosis in 1987 and 2001 in general practice stratified for several background characteristics. Compared to 1987, in 2001 the total incidence rate of dermatophytosis in general practice increased by $18 \%$.
In 2001 versus 1987 the incidence rate of dermatophytosis in general practice increased in young children ( $0-4$ years); infants ( $<1$ year) showed in 2001 a threefold higher incidence rate $(P<0.01)$. The GP more often diagnosed dermatophytosis in girls than boys 2 (not significant for 1987; $P<0.01$ for 2001). In 1987 we found the highest incidence rate in the three big cities and the lowest in the rural

Table 1 Incidence rates per 1,000 person years of all new episodes of dermatophytosis in general practice in 1987 and 2001

\begin{tabular}{|c|c|c|c|c|c|}
\hline & \multicolumn{2}{|l|}{1987} & \multicolumn{2}{|l|}{2001} & \multirow[t]{2}{*}{$P$-value } \\
\hline & Incidence rates & 95\% Confidence intervals & Incidence rates & 95\% Confidence intervals & \\
\hline \multicolumn{6}{|l|}{ Age categories } \\
\hline$<1$ year & 12.8 & $7.5-20.5$ & 35.1 & $29.8-41.1$ & $<0.01$ \\
\hline $1-4$ years & 17.4 & $13.7-21.7$ & 24.8 & $22.6-27.2$ & $<0.01$ \\
\hline $5-9$ years & 19.7 & $16.3-23.7$ & 18.9 & $17.2-20.8$ & 0.69 \\
\hline $10-14$ years & 24.8 & $20.9-29.2$ & 24.0 & $22.1-26.1$ & 0.73 \\
\hline $15-17$ years & 22.7 & $18.5-27.7$ & 27.6 & $24.9-30.6$ & 0.07 \\
\hline Total & 20.8 & $18.9-22.8$ & 24.6 & $23.5-25.7$ & $<0.01$ \\
\hline \multicolumn{6}{|l|}{ Gender } \\
\hline Boys & 20.0 & $17.4-22.8$ & 22.6 & $21.2-24.1$ & 0.08 \\
\hline Girls & 21.7 & $18.9-24.7$ & 26.0 & $24.4-27.6$ & $<0.01$ \\
\hline \multicolumn{6}{|l|}{ Urbanization } \\
\hline$<30,000$ & 16.6 & $13.9-19.8$ & 23.0 & $21.5-24.7$ & $<0.01$ \\
\hline $30,000-50,000$ & 23.3 & $20.0-26.9$ & 18.2 & $16.1-20.5$ & 0.01 \\
\hline$>50,000$ & 20.4 & $16.4-25.1$ & 19.8 & $18.1-21.6$ & 0.80 \\
\hline Big cities $^{\mathrm{a}}$ & 27.7 & $20.8-36.2$ & 24.1 & $20.1-28.7$ & 0.41 \\
\hline \multicolumn{6}{|l|}{ Region } \\
\hline Northern & 14.4 & $10.0-20.0$ & 24.8 & $22.0-27.9$ & $<0.01$ \\
\hline Central & 22.3 & $19.9-24.9$ & 23.9 & $22.5-25.3$ & 0.28 \\
\hline Southern & 19.8 & $16.2-24.0$ & 25.7 & $23.7-27.9$ & $<0.01$ \\
\hline \multicolumn{6}{|l|}{ Season } \\
\hline Winter & 19.3 & $15.6-23.7$ & 22.7 & $20.7-24.9$ & 0.14 \\
\hline Spring & 23.1 & $19.6-27.2$ & 26.4 & $24.2-28.7$ & 0.13 \\
\hline Summer & 19.9 & $16.0-24.5$ & 25.4 & $23.3-27.7$ & 0.02 \\
\hline Autumn & 20.3 & $16.8-24.2$ & 22.2 & $20.3-24.3$ & 0.36 \\
\hline \multicolumn{6}{|l|}{$\mathrm{SES}^{\mathrm{b}}$} \\
\hline Non-manual high & 21.0 & $17.1-25.6$ & 21.9 & $20.0-23.9$ & 0.70 \\
\hline Non-manual middle & 20.0 & $15.1-26.0$ & 24.0 & $21.7-26.5$ & 0.18 \\
\hline Non-manual low and farmers & 23.6 & $14.8-35.8$ & 25.6 & $21.5-30.3$ & 0.71 \\
\hline Manual high/middle & 21.7 & $17.1-27.0$ & 23.4 & $19.1-28.4$ & 0.62 \\
\hline Manual low & 26.7 & $21.2-33.3$ & 25.1 & $21.4-29.3$ & 0.66 \\
\hline \multicolumn{6}{|l|}{ Ethnic origin } \\
\hline Natives and Western immigrants & 21.7 & $19.4-24.1$ & 22.3 & $21.0-23.6$ & 0.65 \\
\hline Non-Western immigrants & 29.9 & $20.9-41.4$ & 33.6 & $29.0-38.7$ & 0.50 \\
\hline
\end{tabular}

a Amsterdam, Rotterdam, The Hague

b According to father's occupation 
areas $(<30,000)$ whereas in 2001 it was distributed more equally over all urbanization levels. Compared to 1987 , in 2001 the incidence rate increased in the rural areas $(<30,000)$ and decreased in small cities $(30,000-50,000)$, whereas it remained stable in larger cities $(>50,000)$ and the three big cities. In 1987 we found the highest incidence rate in the central part of the Netherlands compared to the northern part $(P<0.01)$ whereas in 2001 the incidence rates were not different between regions. In 2001, the incidence rates increased only in the northern and southern part of the Netherlands compared to 1987.

In both surveys the incidence rates were distributed equally over all seasons and SES classes. In 2001 versus 1987 the incidence rate of dermatophytosis in general practice increased in summer $(P=0.02)$.

In 2001, the GP more often diagnosed dermatophytosis in children of non-western immigrants than in children of natives and western immigrants $(P<0.01)$.

\section{Prescriptions}

In 1987 the GPs made 388 prescriptions in the first contact of the episode; in $92.5 \%$ of these episodes only one drug was prescribed. In $10 \%$ of the episodes the GPs did not prescribe any medication in the first contact. During all episodes the GPs made 464 prescriptions resulting in an average prescription rate of 1.16 per episode; in $7.5 \%$ of the episodes the GPs did not prescribe any medication.

In 2001 the GPs made 1,715 prescriptions in the first contact of the episode; in $90.4 \%$ of these episodes only one drug was prescribed. In $22.5 \%$ of the episodes the GPs did not prescribe any medication in the first contact. During all episodes the GPs made 2,333 prescriptions resulting in an average prescription rate of 1.16 per episode; in $18.3 \%$ of the episodes the GPs did not prescribe any medication.

In Table 2 we present the drugs prescribed in the first contact of the episode. In both surveys about three quarters of the dermatophytosis cases were treated with topical drugs; GPs prescribed in about $50 \%$ of the children with dermatophytosis topical antifungals and in about $20 \%$ topical antifungals combined with topical steroids. Oral antifungals were applied in only a very small proportion of the cases.
However, compared to 1987 , in 2001 the proportion of oral antifungal prescriptions almost doubled from $3.3 \%$ to $5.6 \%(P=0.05)$. For oral treatment in 1987 only azoles were prescribed whereas in 2001 both azoles and allylamines were prescribed in almost equal proportions.

\section{Referrals}

In 1987 twelve (2.6\%) and in 2001 thirty-two (1.6\%) of the children with dermatophytosis were referred to the dermatologist. In 1987 the boys to girls ratio of referred children was $3: 1$, whereas in 2001 this was $1: 2$.

\section{Discussion}

Incidence and sociodemographics

The incidence rate of dermatophytosis in general practice increased over the past 14 years which is consistent with a previous study performed in the Netherlands [22]. Considering the decrease of the overall consultation rate of children in Dutch general practice (second Dutch national survey) [14] the increased incidence rate of dermatophytosis in general practice is substantial. Probably our finding is a consequence of an increased prevalence in the population as reported by Sladden et al. [12] who showed that dermatophytosis is becoming a major health problem in the UK and the USA. A previous study reported that the consultation rate for onychomycosis in Dutch general practice increased from 5.9 (1999) to 8.2 (2000-2001) and fell to 4.9 (2002) following a nationwide information campaign performed by the manufacturer of terbinafine in the Netherlands, advising people with onychomycosis to visit their GP [23]. Alternatively the increased incidence rate does not reflect an increase in incidence of dermatophytosis in the population but a higher inclination to present this disease to the GP.

The GPs more often diagnosed dermatophytosis in girls which is different from a previous Dutch study [22]. However, the prevalence of dermatophytosis in the population was found to be higher in boys [3, 4, $6]$ in three studies in Mediterranean countries. Probably girls are more sensitive for the esthetic 
Table 2 Prescriptions in the first contact of episode

\begin{tabular}{llr}
\hline & \multicolumn{2}{l}{ Number $(\%)$} \\
\cline { 2 - 3 } & 1987 & 2001 \\
\hline Total number of episodes & $400(100)^{\mathrm{a}}$ & $2,007(100)^{\mathrm{b}}$ \\
Oral treatments & & \\
Antifungals & $13(3.3)$ & $113(5.6)$ \\
Azoles & $13(3.3)$ & $48(2.4)$ \\
Allylamines & $0(0)$ & $60(3.0)$ \\
Others & $0(0)$ & $5(0.2)$ \\
Topical treatments & & $1,098(54.7)$ \\
Antifungals & $231(57.8)$ & $1,002(49.9)$ \\
Azoles & $209(52.3)$ & $43(2.1)$ \\
Allylamines & $0(0)$ & $41(2.0)$ \\
Undecenoic acid & $20(5.0)$ & $12(0.6)$ \\
Others & $2(0.5)$ & $375(18.7)$ \\
Combinations (antifungals and corticosteroids) & $94(23.5)$ & $20(1.0)$ \\
Corticosteroids & $10(2.5)$ & $31(1.5)$ \\
Emollients & $3(0.8)$ & $78(3.9)$ \\
Others & $37(9.3)$ & $451(22.5)$ \\
No prescription in first contact & $40(10)$ & \\
\hline
\end{tabular}

Prescriptions

aspects of the disease and therefore present this problem more easily to their GP.

The incidence rate increased in rural areas $(<30,000)$ and remained stable in urban areas. A previous study performed in children in rural areas in Turkey [6] reported that the prevalence of dermatophytosis is higher under poor hygienical conditions. However, in the Netherlands, the difference in hygiene conditions between rural and urban areas is negligible. Considering the decrease of the overall consultation rate of children in Dutch general practice (second Dutch national survey) [14] it might reflect an increase in incidence rate of dermatophytosis in children in general practice in urban areas. Children of non-Western immigrants consulted their GP more often with dermatophytosis; in 2001 this difference became more apparent. The significantly higher overall consultation rate in non-western children might explain this difference [14]. The increasing racial and ethnic heterogeneity of the Dutch childhood population might contribute to an increased incidence of dermatophytosis in the population. Previous studies reported that Afro-Caribbean children are particularly affected by tinea capitis in the UK [12] and that the prevalence of tinea capitis in Stockholm increased corresponding with the increased immigration from Africa [24].
Between 1987 and 2001 the prescription pattern of the GP changed. In 2001 more oral antifungals especially terbinafine (not available in 1987) were prescribed. Probably, this was influenced by the nationwide information campaign, as mentioned earlier [23]. In 2001 there were more episodes in which the GP did not prescribe any medication. Probably this has to do with the fact that nowadays more over-the-counter drugs are available for dermatophytosis; patients who initially use these medications may consult their GP in a later phase of the disease which should have consequences for the GPs' management.

The majority of the dermatophytosis patients were treated with topical azoles and a very small proportion with allylamines or other antifungals indicating that dermatophytoses were primarily treated with azoles. This is in accordance with the results of the Cochrane reviews $[15,16]$ and the clinical guideline for the treatment of dermatophytosis issued by the Dutch College of General Practitioners in 1997 [18].

In 2001 there were more episodes that included only one contact with the GP and more oral medication especially allylamines were prescribed. In both surveys the prescription rate per episode is the 
same, but in 2001 there were more episodes in which the GP did not prescribe any medication. The referral rate per episode in 2001 is lower than in 1987. These changes in disease management could be the consequence of the introduction of the clinical guideline for diagnosis and treatment of dermatophytosis issued by the Dutch College of General Practitioners in 1997 [18] which may have improved the care for patients with dermatophytosis in general practice.

\section{Referral}

The boys to girls ratio of referred children concerning dermatophytosis strikingly changed from 3:1 (1987) to $1: 2$ (2001). As suggested earlier, girls may be more sensitive for the esthetic reasons of the disease and therefore consulted the GP more often than boys in 2001. This is supported by our previous analysis [26] showing that girls more often consulted the GP than boys concerning all skin diseases combined. Possibly, for cosmetic reasons girls or their parents put more pressure on the GPs for being referred to a dermatologist.

Strengths and limitations of the study

These two large representative and comprehensive surveys enabled us to assess accurately epidemiological data on dermatophytosis in children. For this study data of only two points in time were available. To identify a sustained trend of the incidence of dermatophytosis in general practice data of multiple points in time are needed.

There were small differences in the design of the two national surveys, which might disturb the comparability of data. For example ICPC coding of the diagnoses was not performed equally in both surveys: in 1987 clerks coded diagnoses afterwards, whereas in 2001 the GPs coded the diagnoses themselves during the consultation. We assume that coding by clerks more often led to a specific diagnostic ICPC code.

In the present study the accuracy of diagnoses (S74) made by the GPs could be subject of debate. The Dutch guideline allows the GP to make the diagnosis of dermatophyte infection in most cases without $\mathrm{KOH}$ preparation or culture [18]. In our analysis we assumed that the diagnoses made by the GPs were correct. In 2001 the participating GPs were trained in coding the diagnoses correctly using ICPC codes. Overall these trained GPs classified diagnoses correctly in about $81 \%$ of the test cases [25].

However, initial analysis showed that the incidence rate of diaper rash (S89) decreased by about $50 \%$ in 2001 [26]. Possibly GPs have coded diaper rash as dermatophytosis which may have lead to an overestimation of the incidence rate of dermatophytosis in 2001. Another possibility is that GPs have coded dermatophytosis as diaper rash in 1987, which may have led to an underestimation of the incidence rate of dermatophytosis in 1987.

\section{Conclusions}

In general practice the reported incidence rate of dermatophytosis in children increased, especially in girls, children of non-western immigrants, rural areas and in the northern and southern part of the Netherlands. This increase in incidence rate could be a consequence of an increasing prevalence in the population. More studies on the population prevalence of dermatophytosis and help seeking behaviour are needed, especially in children of non-western immigrants and rural areas to test this hypothesis.

GPs generally follow the clinical guideline for diagnosis and treatment of dermatophytosis in children which is in accordance with evidence-based knowledge on the effectiveness of different therapies.

Acknowledgements The authors thank all participating GPs and their staff members for providing data. Funding: The Dutch ministry of Health, Welfare and Sports mainly funded the surveys directly or indirectly. In addition, the "Stichting Centraal Fonds RVVZ" contributed financially to the second survey. The analysis reported in this paper was made possible through internal funding of the department of General Practice, Erasmus MC-University Medical Center Rotterdam. Competing interests: All authors declare that they have no competing interests. Authors' contributions: RSAM and JCvdW designed the study. RSAM carried out the analyses and drafted the paper. All authors commented on draft versions and approved the final manuscript.

\section{References}

1. O'Brien JM. Common skin problems of infancy, childhood, and adolescence. Prim Care 1995;22:99-115. 
2. Reichert-Pénétrat $\mathrm{S}$ et al. Epidemiology of dermatophytosis in children living in northeast France: a 5-year study. Pediatr Dermatol 2002;19:103-5.

3. Leibovici V et al. Population-based epidemiologic study of tinea pedis in Israeli children. Pediatr Infect Dis J 2002;21:851-3.

4. Koussidou-Eremondi $\mathrm{T}$ et al. Epidemiology of dermatomycoses in children living in northern Greece 1996-2000. Mycoses 2005;48:11-6.

5. Fuller LC et al. Diagnosis and management of scalp ringworm. Br Med J 2003;326:539-41.

6. Metintas $\mathrm{S}$ et al. Frequency and risk factors of dermatophytosis in students living in rural areas in Eskisehir, Turkey. Mycopathologia 2004;157:379-82.

7. Ogunbiyi AO et al. Prevalence of skin disorders in school children in Ibadan, Nigeria. Pediatr Dermatol 2005;22: 6-10.

8. Fernandes NC et al. Dermatophytoses in children: study of 137 cases. Rev Inst Med Trop Sao Paulo 2001;43:83-5.

9. Bronson DM et al. An epidemic of infection with Trichophyton tonsurans revealed in a 20-year survey of fungal infections in Chicago. J Am Acad Dermatol 1983;8:322-30.

10. Higgins EM et al. Guidelines for the management of tinea capitis. Br J Dermatol 2000;143:53-8.

11. Fuller LC et al. Scalp ringworm in south-east London and an analysis of a cohort of patients from a paediatric dermatology department. Br J Dermatol 2003;148:985-8.

12. Sladden MJ, Johnston GA. Common skin infections in children: clinical review. Br Med J 2004;329:95-9.

13. Aly R. Ecology and epidemiology of dermatophyte infections. J Am Acad Dermatol 1994;31:S21-5.

14. Otters HBM et al. Changing morbidity patterns in Dutch general practice: 1987-2001. Eur J Gen Pract 2005;11:17-22.

15. Crawford $\mathrm{F}$ et al. Topical treatments for fungal infections of the skin and nails of the foot. Cochrane Database Syst
Rev 1999;Issue 3. Art. No.: CD001434. doi: 10.1002/14651858.CD001434.

16. Bell-Syer SEM et al. Oral treatments for fungal infections of the skin of the foot. Cochrane Database Syst Rev 2002; Issue 2. Art. No.: CD003584. doi: 10.1002/14651858.CD003584.17.

17. Williams $\mathrm{H}$ et al. Evidence-based dermatology. London: BMJ Books, 2003.

18. de Kock CA et al. Dermatomycosen. NHG-standaard. [Dermatomycoses. Guideline of the Dutch College of general practitioners]. Huisarts Wet 1997;40:541-52.

19. Bensing JM et al. De nationale studie van ziekten en verrichtingen in de huisartspraktijk. Achtergronden en methoden. [The national survey of diseases and interventions in general practice. Background and methods]. Huisarts Wet 1991;34:51-61.

20. Lamberts H, Wood M. ICPC: International classification of primary care. Oxford: Oxford University Press, 1987.

21. Westert GP et al. Monitoring health inequalities through general practice: the second Dutch national survey of general practice. Eur J Public Health 2005;15:59-65.

22. van de Lisdonk EH et al. Ziekten in de huisartspraktijk. [Diseases in general practice]. 4th ed. Maarssen: Elsevier, 2003.

23. 't Jong GW et al. Marketing in the lay media and prescriptions of terbinafine in primary care: Dutch cohort study. Br Med J 2004;328:931.

24. Hallgren $J$ et al. Increasing tinea capitis prevalence in Stockholm reflects immigration. Med Mycol 2004;42:505-9.

25. van der Linden MW et al. Klachten en aandoeningen in de bevolking en in de huisartspraktijk [Symptoms and diseases in the population and in general practice]. Utrecht/ Bilthoven, NIVEL/RIVM 2004.

26. Mohammedamin RSA et al. Increasing incidence of skin disorders in children? A comparison between 1987 and 2001. BMC Dermatol 2006;6:4. 